\title{
Der Morbus Osgood-Schlatter - eine Diagnose des sportlichen Kindes
}

\author{
Oliver Eberhardt, Thomas Wirth
}

\section{Zusammenfasssung}

Der Morbus Osgood-Schlatter ist eine typische Erkrankung im Jugendalter mit Schmerzsymptomatik im Bereich der Tuberositas tibiae. Betroffen sind meist sportliche Kinder, bei welchen es durch sportliche Aktivitäten mit Sprungbelastungen (Fußball, Basketball, Volleyball) zu Veränderungen der Patellarsehne und der proximalen Tibiaapophyse kommt. Die Therapie orientiert sich an der Schmerzsymptomatik mit Belastungsreduktion, symptomatischer antiphlogistischer Medikation und selten passagerer Ruhigstellung. Die Prognose der Erkrankung ist gut. Fortbestehende Beschwerden unter sportlicher Belastung oder Probleme beim Knien führen nur selten zu einer operativen Therapie. Diese besteht in einer Resektion von Ossikeln und einer Tuberkuloplastik.

\section{Osgood-Schlatter Syndrome - A Diagnosis in the Athletic Child}

Osgood-Schlatter syndrome is a common disease with pain over the tibial tuberosity. Sporting activities that involve jumping and running (e.g. soccer, basketball, volleyball or running) lead to repetitive strain to the tibial tuberosity with traction apophysitis. Treatment depends on the severity of the pain syndrome with modification of sports activities, nonsteroidal inflammatory drugs or immobilisation. The overall prognosis is good with some discomfort in kneeling and activity restriction in some cases. Only in a few cases surgical excision of ossicles and tuberculoplasty may be necessary.

\section{Einleitung}

Der Morbus Osgood-Schlatter ist eine häufige Ursache für Kniegelenkschmerzen im Wachstumsalter. Erstmals wurde die Erkrankung von Robert Osgood und Carl Schlatter 1903 unabhängig voneinander als partielle Avulsion der Tuberositas tibiae beschrieben $[13,17]$. Die Erkrankung tritt v.a. bei sportlich aktiven Jugendlichen auf. Bevorzugt ist das männliche Geschlecht. Der Altersgipfel liegt bei Knaben zwischen dem 13. und 14. und bei Mädchen zwischen dem 10 . und 11 . Lebensjahr. In $25-50 \%$ sind beide Seiten betroffen [19].

Im Folgenden werden Ätiologie, Diagnostik und Therapie in ausführlicher

OP-JOURNAL 2010; 26: 141-144

(c) Georg Thieme Verlag KG Stuttgart · New York DOI http://dx.doi.org/10.1055/s-0030-1250246
Weise besprochen. Insbesondere sollen Hinweise zum Umgang mit sportlichen Jugendlichen mit Morbus OsgoodSchlatter gegeben werden.

\section{Ätiologie}

Die von Ogden und Southwick beschriebene Theorie von Avulsionen der Ossifikationszentren der Tuberositas tibiae ist auch heute noch generell akzeptiert [10, 12]. Ogden beschreibt eine traumatische Lösung eines knorpeligen oder knöchernen Anteils der Tuberositas [11]. Kontrovers diskutiert wird, ob die Pathologie auf Seiten der knöchernen Strukturen oder der Sehne liegt $[16,19]$. Wohl führen repetitive Zugbelastungen an der Patellarsehne zu entzündlichen Veränderungen, welche zu Ossifikationsstörungen der Tuberositas tibiae führen. Neuere Studien sehen die Hauptpathologie im Bereich der Sehnen und der Weichteile. Rosenberg et al. konnten bei
Vergleichen von verschiedenen bildgebenden Verfahren (CT, Szintigrafie, MRT) insultähnliche Veränderungen im Bereich der Patellarsehne feststellen [16]. Auch der Zusammenhang zur Patella alta wurde beschrieben [1]. Im deutschen Sprachraum wird der Morbus Osgood-Schlatter auch in die aseptischen Knochennekrosen eingereiht [6].

\section{Symptomatik}

Die Symptomatik ist geprägt durch Schwellung und Schmerzen im Bereich der distalen Patellarsehne und der Tuberositas tibiae (Abb.1). Die Beschwerden treten v.a. bei sportlichen Aktivitäten mit abrupten Bewegungen und/oder Sprungbelastungen sowie beim treppauf und treppab steigen auf.

Die klinische Einteilung orientiert sich an der Schmerzsymptomatik und der Schmerzdauer. Bewährt hat sich folgende klinische Einteilung [18].

- Grad 1: Schmerzen nach Belastung, welche sich innerhalb von 24 Stunden zurückbilden

- Grad 2: Schmerzen während und nach Belastung, welche die sportliche Aktivität nicht limitieren und sich ebenfalls innerhalb 24 Stunden zurückbilden

- Grad 3: Schmerzen, welche konstant auftreten und sportliche Aktivitäten limitieren

\section{Diagnostik und bildgebende Verfahren}

Schwellung, Schmerzen und Prominenz der Tuberositas tibiae führen zur Diagnose des Morbus Osgood-Schlatter. Bei einer sorgfältigen Anamnese werden vom Patienten Schmerzen unter Belastung und Schmerzreduktion oder Schmerzfreiheit in den sportlichen Ruhephasen beschrieben. Eine Bewegungseinschränkung wird in der Regel ebenso wie eine Ergussbildung nicht beobachtet. Bei der Untersuchung müssen stets andere Pathologien am Kniegelenk ausgeschlossen werden. Die Untersuchung umfasst da- 


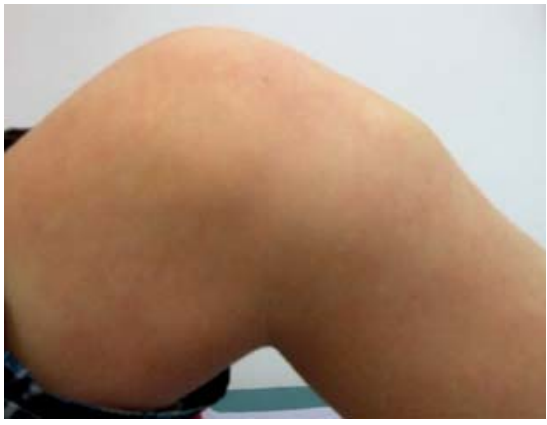

Abb. 1 Prominenz der Tuberositas tibiae bei Morbus Osgood-Schlatter.

bei Meniskustests ebenso wie eine sorgfältige Bandprüfung und die Evaluation der Patellaführung.

Bei Patienten mit akuter Schmerzsymptomatik muss eine Röntgenkontolle erfolgen, um eine Avulsionsfraktur auszuschließen. Im Verlauf sind keine weiteren regelmäßigen Röntgenkontrollen notwendig.

Das Röntgenbild zeigt in der seitlichen Ebene einen aufgelockerten, fragmentierten und verdichteten Apophysenkern. Dabei kann die Form der Fragmentation sehr variabel sein (Abb. $\mathbf{2}$ und $\mathbf{4})$.

Ultraschall und Kernspintomografie können die Diagnose bestätigen, sind jedoch generell nicht notwendig [21]. Fehlen radiologische Zeichen, kann eine MRT-Untersuchung hilfreich sein (Abb. 3). Eine sagittale Spin-Echo-Sequenz zeigt eine vermehrte Signalintensität in der Sehne und eine verminderte Signalintensität im Bereich der knöchernen Strukturen der Tuberositas [16].

\section{Therapie}

\section{Konservative Therapie}

Konservative Maßnahmen bestehen aus medikamentöser, physikalischer und krankengymnastischer Behandlung. Meist werden mehrere einzelne Therapieformen in einem Konzept addiert, um einen größtmöglichen Behandlungserfolg zu erzielen $[2,15]$.

Das Therapiemanagement orientiert sich auch an der o.g. klinischen Einteilung:

Bei Grad 1 und 2, bei welchem die Schmerzsymptomatik nach Beendigung der sportlichen Belastungen komplett rückläufig ist, ist v. a. eine sorgfältige Aufklärung der Eltern über die Erkrankung notwendig. Sportliche Aktivitäten müssen nicht beendet werden. Die Reduk-

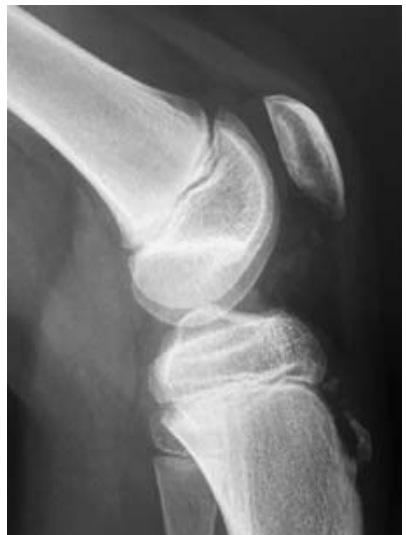

Abb. 2 Tuberositas tibiae mit sekundären Ossifikationszentren bei Morbus Osgood-Schlatter.

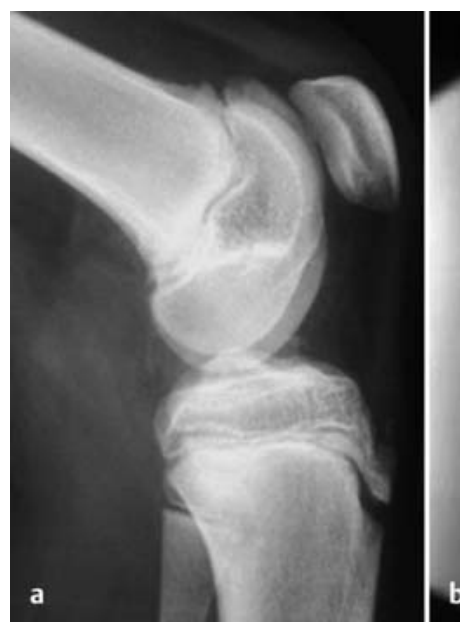

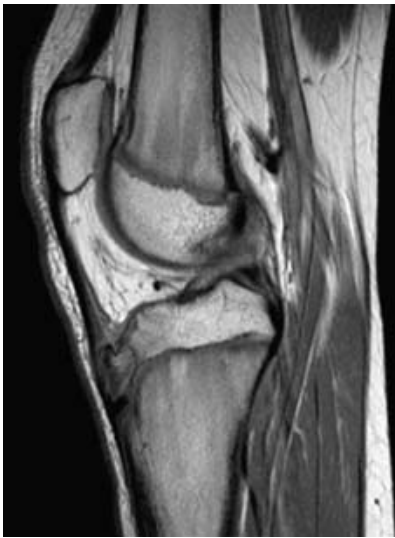

Abb. 3 Kernspintomografie eines Morbus Osgood-Schlatter mit prominentem, zipflig ausgezogenem Apophysenkern und signalintenser Patellarsehne.

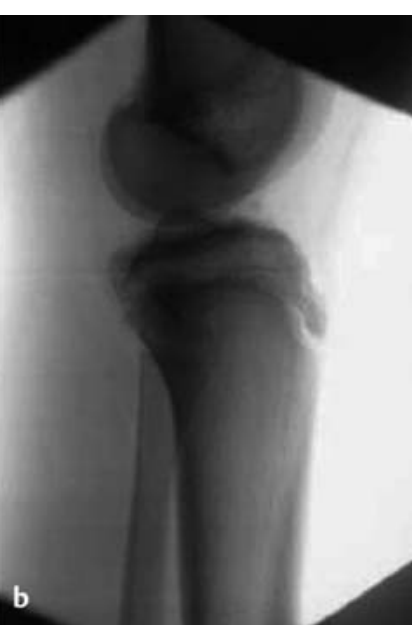

Abb. 4 Morbus Osgood-Schlatter vor und nach Ossikelresektion und Tuberkuloplastik. tion des Umfangs der Belastung kann jedoch sinnvoll sein. Eine Schmerztherapie mit nicht steroidalen Antiphlogistika, Eisbehandlung, unterstützt von Dehnungsübungen für die Hamstrings, kann die Symptomatik reduzieren. Dämpfende Einlagen können den Stress auf die Tuberositas tibiae etwas reduzieren. Lokale Infiltrationen von Kortikoiden sind nicht zu empfehlen.

Bei Grad 3, wenn Schmerzen nicht mehr zwischen sportlichen Belastungen verschwinden, sind Sportpausen und eine Ruhigstellung in einem Gips notwendig. Ein spezifisches Rehabilitationsprogramm mit Rückführung in den Wettkampfsport ist in Abhängigkeit vom Aktivitätsniveau des jungen Sportlers notwendig.

Krankengymnastisches und physikalisches Rehabilitationsprogramm [21]

\section{Phase 1}

Der Beginn einer konservativen Therapie besteht zunächst aus einer Reduktion der
Belastung, wenn notwendig sogar aus einer zeitweisen Sportkarenz, die individuell bestimmt werden muss. NSAR, Eis, passagere Ruhigstellung in einer Orthese oder gar einem Gipstutor müssen entsprechend der Symptomatik angepasst werden. Eine Mobilisation an Unterarmgehstützen unter einer Teilbelastung ist oft für kurze Zeit notwendig. Sportliche Aktivitäten, bei welchen repetitive Zugbelastungen an der Patellarsehne auftreten, müssen vermieden werden. Hierzu gehören Sportarten mit abrupten Stoppund Sprintbewegungen sowie Sportarten mit Sprungbelastungen. Auch Lauftraining sollte zunächst vermieden werden.

\section{Phase 2}

Bei Erreichen einer Schmerzfreiheit können spezifische Übungen durchgeführt werden. Sofern eine Entlastung erfolgt ist, sollte ein vorsichtiger Belastungsaufbau erfolgen. Eine Steigerung von $10 \mathrm{~kg} /$ Woche kann empfohlen werden, wobei dies im schmerzfreien Rahmen gesche- 
hen sollte. Im Vordergrund stehen zunächst Stretchingübungen für den Rectus femoris, die Hamstrings, den Tractus iliotibialis. Exzessive Flexionsübungen sollten am Anfang noch vermieden werden. Zu den Dehnungsübungen kommen nun Kräftigungen hinzu. Isometrische Anspannungen folgen, Beinpresse mit leichten Gewichten und Übungen auf dem Stepper. Propriozeptive Übungen sollten integriert werden.

Phase 3

An Phase 2 schließt sich ein Walking/ Running-Programm an. Am Anfang sollte zunächst nur Walking in einem altersangepassten Zeitraum erfolgen. Erst bei beschwerdefreiem Gehen kann mit Joggingübungen begonnen werden. Bei schmerzfreiem Joggen können dann abrupte Stopp- und Sprintübungen erfolgen, ehe die volle sportliche Aktivität aufgenommen werden kann und das sportartspezifische Training beginnt.

\section{Operative Therapie}

\section{Ossikelresektion und Tuberkuloplastik}

Einige Patienten entwickeln trotz konsequenter Schonung und konservativer Therapie schmerzhafte Ossikel im Bereich der Tuberositas tibiae. Smith empfiehlt neben einem Entfernen der Ossikel auch eine Tuberkuloplastik. Die Operation erfolgt in Rückenlage. Eine s-förmige Inzision oder ein Längsschnitt über der Tuberositas tibiae von $5 \mathrm{~cm}$ wird empfohlen $[14,18]$. Alternativ zur Längsinzision der Patellarsehne kann eine quere Inzision $1 \mathrm{~cm}$ oberhalb der Tuberositas tibiae erfolgen [14]. Das Paratenon wird in Längsrichtung parallel zur Patellarsehne inzidiert. Die Ossikel werden ausgelöst und entfernt. Eine distale Längsinzision der Patellarsehne kann für die Tuberkuloplastik notwendig werden. Mit einem gebogenen Osteom (6-9 mm) wird die Tuberositas tibiae abgerundet (Abb.4). 2-3 invertierende, tiefe Nähte verschließen die Patellarsehne. Nach subkutaner Naht kann eine intrakutane Hautnaht durchgeführt werden.

Die postoperative Nachbehandlung erfolgt zunächst mit einer Teilbelastung von $20 \mathrm{~kg}$. Ein Bewegungslimit für 2 Wochen mit $60^{\circ}$ Flexion wird empfohlen. Der Übergang zur Vollbelastung erfolgt nach 4 Wochen. Eine Sportkarenz sollte für 8-12 Wochen eingehalten werden. In der postoperativen Phase kann bereits mit dem krankengymnastischen und physikalischen Rehabilitationsprogramm begonnen werden.

\section{Diskussion}

Der Morbus Osgood-Schlatter ist eine häufige Ursache des Knieschmerzes beim sportlich aktiven Jugendlichen. Zeitgleich wurde die Erkrankung von Osgood und Schlatter 1903 beschrieben $[13,17]$. Die Ursache der Erkrankung wurde von beiden in einer Avulsionsverletzung gesehen.

Ständige Zugbelastung an der Patellarsehne im Wachstumsschub und repetitive Mikrotraumata führen zu einem gestörten Wachstum im Bereich der noch knorpeligen Apophyse $[12,13]$.

Derzeit ist noch nicht geklärt, ob es sich nicht doch um eine Tendinitis mit konsekutiver knöcherner Pathologie handelt. Beim Vergleich von Szintigrafie, MRT und Röntgen konnten Rosenberg et al. bei allen Patienten Weichteilveränderungen an der Patellarsehne finden. Dabei handelte es sich im MRT um Verdickungen der Patellarsehne und Signalveränderungen in der Sehne selbst. Währenddessen wurden nur bei $32 \%$ knöcherne Veränderungen gesehen. Die Ursache des Morbus Osgood-Schlatter wird nach den Erkenntnissen von Rosenberg et al. in einem Insult der Patellarsehne gesehen [16].

In der Literatur wird beschrieben, dass die Erkrankung überwiegend bei Knaben auftritt. Unter Berücksichtigung verschiedener Sportarten mit entsprechender Geschlechtsverteilung ist der Anteil von weiblichen Betroffenen eher gleich.

Der Altersgipfel liegt bei Knaben zwischen dem 13. und 14. und bei Mädchen zwischen dem 10. und 11. Lebensjahr [18].

Das klinische Bild ist geprägt durch Schwellung und Schmerzen. Die Beschwerden treten v.a. bei oder nach sportlichen Aktivitäten auf. Vor allem beim Laufen, Springen und Treppensteigen, auf- und abwärts, kommt es zur typischen Schmerzsymptomatik im Bereich der Tuberositas tibiae. Hauptsächlich betroffen sind sportlich aktive Jugendliche. Prädisponierende Sportarten sind Fußball und Basketball. Weiterhin tritt der Morbus Osgood-Schlatter bei Sportlern mit repetitiven Sprungbelastungen, wie sie beim Turnen und beim Ballett typisch sind, auf $[15,18,21]$.
Im Verlauf der Erkrankung kommt es häufig zu einer Fragmentation der Apophyse. Radiologische Auffälligkeiten können jedoch auch vollständig fehlen [21]. Als prädisponierender Faktor wurde auch die Patella alta angesehen [1]. Dies konnte von Pihlajamäki et al. nicht bestätigt werden [14]. Eine elongierte Patellarsehne wurde bei Patienten mit Morbus Osgood-Schlatter von Visuri et al. beschrieben [20].

Die Therapie orientiert sich an der vorhandenen Beschwerdesymptomatik. Hilfreich ist die Einteilung in Schweregrade. Grad 1 mit Schmerzen nach Belastung, welche sich innerhalb 24 Stunden zurückbilden. Grad 2 mit Schmerzen während und nach Belastung, welche die sportliche Aktivität nicht limitieren und sich innerhalb 24 Stunden zurückbilden. Und Grad 3 mit Schmerzen, welche konstant auftreten und sportliche Aktivitäten limitieren.

In einer finnischen Studie konnte der Zusammenhang zwischen vermehrter sportlicher Aktivität und Auftreten des Osgood-Schlatter nachgewiesen werden. So zeigten sich bei sportlich aktiven Individuen in $21,2 \%$ und bei nicht sportlich aktiven Individuen in nur 4,5\% der Fälle Veränderungen im Sinne eines Morbus Osgood-Schlatter [8].

Sportliche Aktivitäten, bei welchen repetitive Zugbelastungen an der Patellarsehne auftreten, sind alle Sportarten mit abrupten Stopp- und Sprintbewegungen sowie Sportarten mit Sprungbelastungen. Demnach sind Sportarten wie Fußball, Basketball, Handball, Volleyball sowie Ballett, aber auch Sprungdisziplinen bei Leichathletik eher das Knie belastend und damit prädisponierend für einen Morbus Osgood-Schlatter als Schwimmen und Fahrradfahren. Bei diesen Sportarten kommt es in der Regel nicht zu derartigen Zugbelastungen an der Patellarsehne, welche $\mathrm{zu}$ einem Morbus Osgood-Schlatter führen. Auch regelmäBiges Joggen auf weichem Untergrund führt nicht zu vermehrten Zugbelastungen an der Patellarsehne.

Bei einem symptomatischen Kind oder Jugendlichen stellt sich für den Orthopäden, Kinderorthopäden und Sportmediziner nicht die grundsätzliche Frage „Welche Sportart darf der Betroffene noch durchführen?" Vielmehr müssen das sportliche Aktivitätsniveau des Kindes oder des Jugendlichen und die vorhandene Beschwerdesymptomatik individuell beurteilt werden. 
Ein aufgetretener Morbus Osgood-Schlatter bedeutet nicht das Ende des Fußballspielens. Handelt es sich um einen Schweregrad 1 und 2, so reicht eine Elternaufklärung über den Befund und die Problematik häufig aus. Bei Schmerzen, die sich nach 24 Stunden vollständig zurückbilden, sind nur kleinere Sportpausen notwendig. Eine längere Sportaufgabe ist nicht zu empfehlen, da bei Beginn erneuter sportlicher Aktivitäten meistens mehr Probleme auftreten. Ein Sportartenwechsel muss wohlüberlegt sein, da die Kinder und Jugendlichen mit ihrer Disziplin zumeist fest verbunden sind. Eine Einlagenversorgung mit dämpfenden, schockabsorbierenden Materialien kann häufig hilfreich sein.

Handelt es sich um einen Schweregrad 3, treten Beschwerden regelmäßig auch im alltäglichen Leben auf und limitieren diese sportliche Aktivitäten, so muss auch hier die Sportart nicht sofort gewechselt werden. Ein Therapieregime mit Eistherapie, Hamstrings und Quadrizepsstretching, evtl. 3-4-wöchiger Ruhigstellung (OS Gipstutor) führt häufig zur Beschwerdebesserung und erlaubt die Wiederaufnahme der Sportart. Dies sollte auch dem Kind und Jugendlichen nicht verwehrt werden.

Bei Kadern und Leistungssportlern muss sicherlich ein stufenweises Rehabilitationsprogramm erarbeitet werden, bevor die Jugendlichen wieder die volle Belastbarkeit erreichen. Dies ist wichtig, um erneuten Exazerbationen der Symptomatik Vorbeugung zu leisten. Entsprechende Übungseinheiten für den Morbus Osgood-Schlatter sind in ausführlicher Weise in Micheli und Kocher's The Pediatric and Adolescent Knee beschrieben $[2,9,21]$.

Das Knie weniger belastende Sportarten sind Schwimmen und Radfahren. Die Empfehlung oder Vermeidung verschiedener sportlicher Aktivitäten und Sportarten orientiert sich jedoch immer am betroffenen Sportler. Allgemeingültige Empfehlungen zu Sportarten beim Morbus Osgood-Schlatter sind daher nur bedingt möglich.

Lokale Infiltrationen mit Lokalanästhetika sind in der Literatur zur Schmerzreduktion beschrieben [16]. Aus unserer Sicht sollte jedoch nicht vor sportlichen Aktivitäten eine Injektion erfolgen, da eine Exazerbation oder eine Verschlechterung der Symptomatik begünstigt wer- den kann. Eine lokale Infiltration mit Kortikoiden wird nicht empfohlen [21].

Die Prognose der Erkrankung ist gut. Der Morbus Osgood-Schlatter ist in der Regel selbstlimitierend. Nach 12-24 Monaten kommt es, meist bei Wachstumsabschluss, zu einer spontanen Besserung. Bis zu $90 \%$ der Patienten können mit konservativer Therapie, Eisbehandlung, Aktivitätsänderung, Sportreduktion oder passagerer Ruhigstellung behandelt werden [4].

Ein kleiner Anteil von Patienten entwickelt ein schmerzhaftes Ossikel, welches chirurgisch entfernt werden kann. 24\% leiden an geringen Einschränkungen bei sportlichen Aktivitäten. Krause et al. beschrieben bei $60 \%$ im Verlauf persistierende Probleme beim Knien. In einer großen Serie von Pihlajamäki et al. konnte diese große Anzahl etwas relativiert werden. Bei 178 Patienten zeigten sich jedoch nach operativer Therapie noch bei 39\% Beschwerden beim Knien [7]. Als seltene Komplikation ist das Genu recurvatum bei vorzeitigem Fugenverschluss in einzelnen Fällen beschrieben [22].

Bei konservativ nicht beherrschbarer Symptomatik besteht die operative Therapie aus einer Resektion der störenden Ossikel. Gleichzeitig sollte jedoch eine Tuberkuloplastik der Tuberositas tibiae erfolgen. In mehreren Studien konnte gezeigt werden, dass bei gleichzeitiger Tuberkuloplastik die Erfolgsrate höher war $[3,5,14]$. Flowers und Bhadreshwar beschrieben mit diesem Vorgehen bei 95\% in einem 5-Jahres-Nachuntersuchungszeitraum eine Schmerzfreiheit [3].

Zusammengefasst kann festgestellt werden, dass der Morbus Osgood-Schlatter eine gutartige, selbstlimitierende Erkrankung ist. Sie tritt häufig bei Fußball-, Basketballspielern, bei Sprungsportarten und beim Ballett auf. Konservative Maßnahmen führen in der Regel zu Schmerzreduktion und Ausheilung der Erkrankung. Nur in wenigen Fällen mit Bildung von Ossikeln ist eine operative Therapie notwendig.

\section{Literatur}

${ }^{1}$ Aparicio G, Abril J, Calvo E et al. Radiological study of patellar height in Osgood-Schlatter disease. J Pediatr Orthop 1997; 17: 63-66

2 Faigenbaum AD, Westcott WL, Loud RL et al. The effects of different resistance training protocols on muscular and endurance development in children. Pediatrics $1999 ; 104: 1-7$
${ }^{3}$ Flowers MJ, Bhadreshwar DR. Tibial tuberosity excision for symptomatic Osgood-Schlatter disease. J Ped Orthop 1995; 15: 292-297

${ }^{4}$ Gholve PA, Scher DM, Khakharia S et al. Osgood Schlatter syndrome. Curr Opin Pediatr 2007; 19: 44-50

${ }^{5}$ Glynn MK, Regan BF. Surgical treatment of Osgood-Schlatter's disease. J Pediatr Orthop 1983; 3: 216-219

${ }^{6}$ Hefti F. Kinderorthopädie in der Praxis. Heidelberg: Spinger; 2006: 289-290

7 Krause BL, Williams JP, Catterall A. Natural history of Osgood-Schlatter disease. J Pediatr Orthop 1990; 10: 65-68

${ }^{8}$ Kujala UA, Kvist M, Heinonen O. OsgoodSchlatter's disease in adolescent athletes. A retrospective study of incidence and duration. Am J Sports Med 1985; 13: 236-241

${ }^{9}$ Metcalf JA, Roberts SO. Strength training and the immature athlete: an overview. Pediatr Nurs 1993; 19: 325-332

${ }^{10}$ Mital MA, Matza RA, Cohen J. The so-called unresolved Osgood-Schlatter lesion: a concept based on fifteen surgically treated lesions. J Bone Joint Surg [Am] 1980; 62: 732-739

11 Ogden JA. Skeletal injury in the child. Philadelphia: Lea \& Ferbiger; 1992: 814-815

12 Ogden JA, Southwick WO. Osgood-Schlatter's disease and tibial tuberosity development. Clin Orthop Relat Res 1976; 116: 180-189

${ }^{13}$ Osgood RB. Lesions of the tibial tubercle occuring during adolescence. Boston Med Surg J1903; 148: 114-117

14 Pihlajamäki HK, Mattila VM, Parviainen $M$ et al. Long-term outcome after surgical treatment of unresolved Osgood-Schlatter disease in young men. J Bone Joint Surg [Am] 2009; 91: 2350-2358

15 Renström PA. Sportverletzungen und Überlastungsschäden. Köln: Deutscher Ärzte-Verlag; 1997

16 Rosenberg ZS, Kawelblum M, Cheung YY et al. Osgood-Schlatter lesion: fracture or tendinitis? Scintigraphic, CT and MR imaging features. Radiology 1992; 185: 853-858

17 Schlatter C. Verletzungen des schnabelförmigen Fortsatzes der oberen Tibiaepiphyse. Beitr klin Chir 1903; 38: 874-887

${ }^{18}$ Smith $A D$. Osgood-Schlatter Disorders and related Extensor Mechanism Problems. In: Micheli LJ, Kocher MS, eds. The pediatric and adolescent Knee. Philadelphia: Saunders; 2006

19 Tachdjian OM. Pediatric orthopaedics. 4th ed. Philadelphia: Saunders; 2009

20 Visuri T, Pihlajamäki HK, Mattila VM et al. Elongated patellae at the final stage of Osgood Schlatter disease: a radiographic study. Knee 2007; 14: 198-203

${ }^{21}$ Wong J, Maffulli N. Tendinopathy of the Extensor Apparatus of the Knee. In: Micheli LJ, Kocher MS, eds. The pediatric and adolescent Knee. Philadelphia: Saunders; 2006

22 Zimbler S, Merkow S. Genu recurvatum: a possible complication after Osgood-Schlatter disease. A case report. J Bone Joint Surg [Am] 1984; 66: 1129-1130

\section{Dr. med. Oliver Eberhardt \\ Oberarzt Orthopädie}

Prof. Dr. med. Thomas Wirth

Ärztlicher Direktor Orthopädie

Orthopädische Klinik

Klinikum Stuttgart, Olgahospital

Bismarckstraße 8, 70176 Stuttgart

o.eberhardt@klinikum-stuttgart.de 